\title{
Development and Validation of a Robust LC-MS/MS Method for the Simultaneous Quantification of Doxifluridine and its Two Metabolites in Beagle Dog Plasma
}

\author{
In-hwan Baek, Jung-woo Chae, Han Jung Chae, ${ }^{\dagger}$ and Kwang-il Kwon* \\ College of Pharmacy, Chungnam National University, Daejeon 305-764, Korea. *E-mail: kwon@cnu.ac.kr \\ ${ }^{\dagger}$ College of Medicine, Chonbuk National University, Jun-ju 561-180, Korea \\ Received March 23, 2010, Accepted June 19, 2010
}

\begin{abstract}
A reverse-phase HPLC method with detection by mass spectrometry is described for the simultaneous determination of doxifluridine and its two active metabolites, 5-fluorouracil (5-FU) and 5-fluorouridine (5-FUrd), in beagle dog plasma. The optimal chromatographic separation was achieved on a Waters Xterra ${ }^{\circledR} \mathrm{C}_{18}$ column $(4.6 \times 250 \mathrm{~mm}$ i.d., $5 \mu \mathrm{m}$ particle size) with a mobile phase of $0.1 \%$ formic acid in a mixture of $99 \%$ methanol and purified water $(99: 1$, $\mathrm{v} / \mathrm{v})$. The developed method was validated in beagle dog plasma with a lowest limit of quantification of $0.05 \mu \mathrm{g} / \mathrm{mL}$ for both doxifluridine and 5-FU, and $0.2 \mu \mathrm{g} / \mathrm{mL}$ for 5-FUrd. Doxifluridine and its two metabolites were stable under the analysis conditions, and intra- and inter-day accuracies exceeded $92.87 \%$, with a precision variability $\leq 11.34 \%$ for each analyte. Additionally, the method for quantifying doxifluridine and its two metabolites, 5-FU and 5-FUrd, in beagle dog plasma was applied successfully to the analysis of pharmacokinetic samples.
\end{abstract}

Key Words: Doxifluridine, 5-Fluorouracil, 5-Fluorouridine, LC-MS/MS, Beagle dog

\section{Introduction}

Doxifluridine (5'-deoxy-5-fluorouridine, 5 '-dFUR) is an oral prodrug of 5-fluorouracil (5-FU) that is converted to the cytotoxic agent 5-FU in the presence of thymidine phosphorylase. ${ }^{1,2}$ $5-\mathrm{FU}$ is an antineoplastic agent that is used alone or in combination for the chemotherapy of advanced gastrointestinal cancer, breast cancer, and several other types of cancer. ${ }^{3}$ Doxifluridine was developed to improve the therapeutic index of 5-FU and to reduce its toxicity. ${ }^{2}$

Doxifluridine is converted to 5-FU by thymidine phosphorylase (Fig. 1), which activity increases in tumor tissue. ${ }^{4,5}$ In the body, 5-FU is metabolized rapidly, particularly in the liver, to give various metabolites with well-known antineoplastic properties. ${ }^{6}$ Among them, 5-FU is cleared enzymatically from plasma to produce 5-fluorouridine (5-FUrd), which is the rate-limiting step catalyzed by uridine phosphorylase. ${ }^{7}$ 5-FU and 5-FUrd have antineoplastic activity, but doxifluridine is not directly cytotoxic itself. ${ }^{3,8}$ The simultaneous pharmacokinetic study of doxifluridine, 5-FU and 5-FUrd are important for evaluation of optimal dosage regimen of doxifluridine, and the analytical method for simultaneous determination of doxifluridine, 5-FU, and 5-FUrd would be useful for the pharmacokinetic study of doxifluridine.

Several high-performance liquid chromatography (HPLC) methods have been developed for the analysis of doxifluridine, 5-FU, or its nucleosides including 5-FUrd. However, no study has demonstrated a HPLC with tandem mass spectrometry (HPLC-MS/MS) method for the simultaneous determination of doxifluridine, 5-FU, and 5-FUrd. Woo et al. reported a LCMS/MS method that quantifies doxifluridine and 5-FU. ${ }^{2}$ Joulia et al. developed a HPLC-ultraviolet method that allows the simultaneous determination of 5-FU, 5-FUrd, and 5-fluoro-2'deoxyuridine (5-FdUrd), but it requires complex LC conditions with relatively long analysis times. ${ }^{3}$

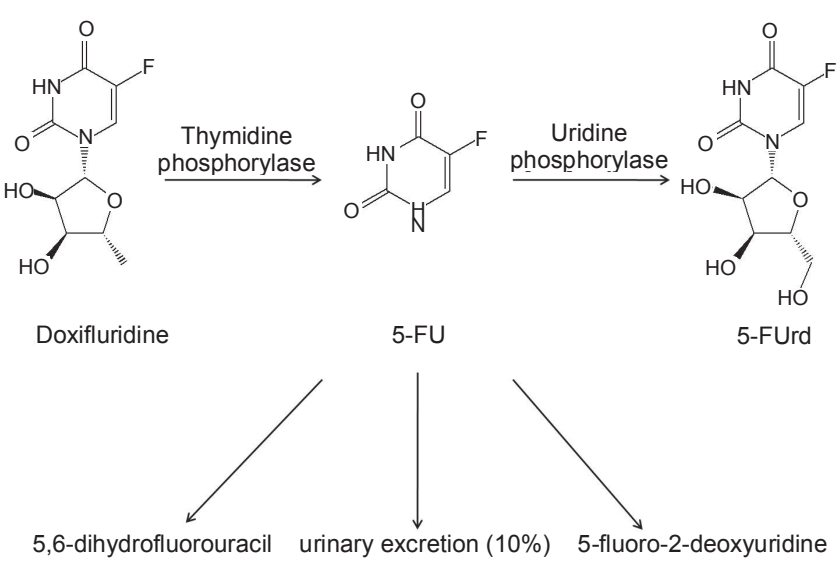

Figure 1. Bioactivation pathway of doxifluridine.

The aim of this work was to develop a selective and sensitive HPLC-MS/MS method for the simultaneous quantification of doxifluridine, 5-FU, and 5-FUrd in beagle dog plasma. The method has been fully validated and has been applied to a pharmacokinetic study of doxifluridine in beagle dogs.

\section{Experimental}

Chemicals and reagents. Doxifluridine of $>99 \%$ purity was supplied by Dong A Pharmaceutical Co. Ltd. (Seoul, Korea). The 5-FU, 5-FUrd, and 5-chlorouracil (internal standard, IS) were obtained from Sigma Chemical Co. (St. Louis, MO, USA). HPLC-grade methanol (MeOH) was purchased from Merck Co. (Darmstadt, Germany) and ethyl acetate was obtained from J.T. Baker (Philipsburg, MT, USA). All other chemicals and solvents were of the highest analytical grade available.

Standard and quality-control samples. Primary stock solutions of doxifluridine, 5-FU, and 5-FUrd for the standard and 
quality control (QC) samples were prepared by weighing separately. The primary stock solutions $(1 \mathrm{mg} / \mathrm{mL})$ of doxifluridine, 5-FU, 5-FUrd, and the IS (5-chlorouracil) were prepared in $\mathrm{MeOH}$ and stored at $-20{ }^{\circ} \mathrm{C}$. Separate working solutions were used to prepare calibration standards of doxifluridine, and its metabolites and were further diluted in $\mathrm{MeOH}$ to yield concentrations of $0.5,2,10,30,75$, and $100 \mu \mathrm{g} / \mathrm{mL}$ doxifluridine; 0.5 , $2,10,20,30$, and $50 \mu \mathrm{g} / \mathrm{mL} 5-\mathrm{FU}$; and 2, 5, 20, 50, 75, and 100 $\mu \mathrm{g} / \mathrm{mL} 5$-FUrd. The IS working solution $(5 \mu \mathrm{g} / \mathrm{mL})$ was prepared by diluting IS stock solution $(1 \mathrm{mg} / \mathrm{mL})$ with $100 \% \mathrm{MeOH}$.

The calibration standards were prepared fresh by spiking $30 \mu \mathrm{L}$ of the three standard working solutions, doxifluridine, 5-FU, 5-FUrd, respectively, into beagle dog plasma $(210 \mu \mathrm{L})$ at a ratio of 1:10. Calibration standards containing doxifluridine and its metabolites were prepared fresh at concentrations of $0.05,0.2,1,3,7.5$, and $10 \mu \mathrm{g} / \mathrm{mL}$ doxifluridine; $0.05,0.2,1,2,3$, and $5 \mu \mathrm{g} / \mathrm{mL} 5-\mathrm{FU}$; and $0.2,0.5,2,5,7.5$, and $10 \mu \mathrm{g} / \mathrm{mL} 5$-FUrd. QC samples were prepared at concentrations of $0.05,0.2,7.5$, and $10 \mu \mathrm{g} / \mathrm{mL}$ doxifluridine; $0.05,0.2,3$, and $5 \mu \mathrm{g} / \mathrm{mL} \mathrm{5-FU;} \mathrm{and}$ $0.2,0.5,7.5$, and $10 \mu \mathrm{g} / \mathrm{mL} 5$-FUrd by diluting the working solutions for QC samples in control beagle dog plasma. The calibration standards and QC samples were vortexed for about $30 \mathrm{~s}$ before processing.

Sample preparation. Plasma calibration standards, plasma blanks, and QC samples $(300 \mu \mathrm{L})$ were added to $1 \mathrm{~mL}$ of $0.05 \mathrm{M}$ $\mathrm{HCl}$ solution with $30 \mu \mathrm{L}$ of IS (5-chlorouracil) spiking solution. For liquid/liquid extraction of doxifluridine, its metabolites, and the IS, $5 \mathrm{~mL}$ of ethyl acetate was added and mixed using a rotator for $10 \mathrm{~min}$. After centrifugation at $3880 \times \mathrm{g}$ for $10 \mathrm{~min}$ at ambient temperature, the supernatant was transferred and evaporated to dryness under a nitrogen stream at $40{ }^{\circ} \mathrm{C}$. The dried extract was resuspended in $100 \mu \mathrm{L}$ of $\mathrm{MeOH}$, and then $10 \mu \mathrm{L}$ was analyzed by HPLC subjected to LC-MS/MS.

Chromatography. An 1100 series liquid chromatography system (Agilent Technologies, Santa Clara, CA, USA) consisting of a solvent degasser, binary pumps, an autosampler, a colu$\mathrm{mn}$ heater, and reversed-phase column $\left(\right.$ Xterra ${ }^{\circledR} \mathrm{C}_{18}, 4.6 \times 250$ mm i.d., $5 \mu \mathrm{m}$ particle size; Waters, Milford, MA, USA) was used for the chromatographic separation of doxifluridine, 5-FU, 5 -FUrd, and the IS. The isocratic mobile phase contained $0.1 \%$ formic acid in a mixture of $\mathrm{MeOH}$ and purified water (99:1, $\mathrm{v} / \mathrm{v}$ ) at a flow rate of $0.3 \mathrm{~mL} / \mathrm{min}$. The column and autosampler tray temperatures were maintained at $25{ }^{\circ} \mathrm{C}$ and $4{ }^{\circ} \mathrm{C}$, respectively.

Mass spectrometry. The plasma concentrations of doxifluridine, 5-FU, and 5-FUrd were quantified by LC-MS using a PE SCIEX API 2000 (triple-quadrupole) LC-MS/MS system (Applied Biosystems, Foster City, CA, USA) equipped with an electrospray ionization interface to generate negative ions. The turbo-ion spray interface was operated in the negative ion mode at $5500 \mathrm{~V}$ and $350{ }^{\circ} \mathrm{C}$. The operating conditions, optimized with a flow injection of a mixture of all analytes, were as follows: exhausting gas, 45 psi; nebulizing gas, 95 psi; curtain gas, 50 psi; orifice voltage (declustering potential), $-36.3 \mathrm{~V}$ for doxifluridine, $-26.0 \mathrm{~V}$ for $5-\mathrm{FU},-48.0 \mathrm{~V}$ for $5-\mathrm{FUrd}$, and $-31 \mathrm{~V}$ for IS; ring voltage (focusing potential), $-380 \mathrm{~V}$ for doxifluridine, -330 $\mathrm{V}$ for $5-\mathrm{FU},-320 \mathrm{~V}$ for $5-\mathrm{FUrd}$, and $-330 \mathrm{~V}$ for IS; collision energy (CE), $-48.3 \mathrm{~V}$ for doxifluridine, $-26 \mathrm{~V}$ for $5-\mathrm{FU},-22 \mathrm{~V}$ for 5-FUrd, and-30 V for IS. Quantification was performed by multiple reaction monitoring (MRM) of the deprotonated precursor ions and the related product ions for doxifluridine, 5-FU, and 5-FUrd using an IS method with peak area ratios. The mass transitions used for doxifluridine, 5-FU, 5-FUrd, and the IS were $m / z 244.82 \rightarrow 41.90,128.82 \rightarrow 41.24,260.87 \rightarrow 128.42$, and $144.76 \rightarrow 41.20$, respectively. The dwell time for each transition was $300 \mathrm{~ms}$, quadrupoles Q1 and Q3 were set at unit resolution, and the analytical data were processed using Analyst 1.4.1 software (Applied Biosystems)

Method validation. To validate the assay, tests were performed on the specificity, calibration curve, intra- and inter-assay precision, intra- and inter-assay accuracy, extraction recovery, matrix effect, plasma sample stability under five conditions, and stock solution stability.

The specificity of the method was determined by analyzing five different batches of beagle dog plasma to demonstrate the lack of chromatographic interference from endogenous plasma components. A calibration curve was generated from the standard solutions to confirm a linear relationship between the peak area ratio and the concentration of doxifluridine. The slope, intercept, and coefficient of determination $\left(r^{2}\right)$ were calculated as regression parameters of a weighted $\left(1 / y^{2}\right)$ linear regression. The same evaluation protocols were followed to test the specificity for 5-FU and 5-FUrd.

Five validation batches were examined to assess the intraand inter-assay precision and accuracy of the method. ${ }^{9}$ Each batch was processed on a separate day with five set of calibration samples and five replicates of QC samples at 0.05, 0.2, 3, and $5 \mu \mathrm{g} / \mathrm{mL}$ doxifluridine; $0.05,0.2,3$, and $5 \mu \mathrm{g} / \mathrm{mL} 5-\mathrm{FU}$; and $0.2,0.5,7.5$, and $10 \mu \mathrm{g} / \mathrm{mL} 5$-FUrd. Accuracy was determined as the percentage difference of the measured concentration from the nominal concentration, and the coefficient of variation (CV) was used to report the precision. ${ }^{10}$

The recovery of doxifluridine, 5-FU, and 5-FUrd was determined by comparing the response of the analytes extracted from replicate QC samples $(n=3)$ at low, medium, and high QC with the response of analytes from post-extracted plasma standard samples at the equivalent concentration. ${ }^{11}$ The matrix effect of plasma constituents over the ionization of analytes was determined by comparing the responses of analytes from neat samples at equivalent concentrations. ${ }^{12}$ The matrix effect was determined at the same concentrations for each analyte as in the recovery experiment. The recovery and matrix effect of the IS was determined at $0.5 \mu \mathrm{g} / \mathrm{mL}$.

Drug-free control plasma samples were spiked with low OC $(0.05 \mu \mathrm{g} / \mathrm{mL})$ and high QC $(10 \mu \mathrm{g} / \mathrm{mL})$ doxifluridine to study its stability in plasma. Short-term, post-extraction, freeze-thaw, and long-term stability was assessed. ${ }^{8}$ To determine short-term stability, samples were analyzed after $6 \mathrm{~h}$ at room temperature; long-term stability was determined after 75 days of storage at $-70{ }^{\circ} \mathrm{C}$. The stability of doxifluridine in extracts was examined after $24 \mathrm{~h}$ of storage at $4{ }^{\circ} \mathrm{C}$. The freeze-thaw stability was evaluated by analyzing the samples after three freeze-thaw cycles $\left(-70{ }^{\circ} \mathrm{C}\right.$ to room temperature). The same evaluations were performed for 5-FU and 5-FUrd. The stability of the stock solutions of doxifluridine, 5-FU, and 5-FUrd were examined after 75 days at $-20{ }^{\circ} \mathrm{C}$. 
Animal study. Seven 13-month-old male beagle dogs weighing $12.55 \pm 1.07 \mathrm{~kg}$ (Central Laboratory Animal Inc., Seoul, Korea) were used for the pharmacokinetic study. They were housed individually in stainless-steel cages in a controlled environment (temperature, $18.0-24.0{ }^{\circ} \mathrm{C}$; humidity, 30.0 - 70.0\%; $12 \mathrm{~h}$ light $/ 12 \mathrm{~h}$ dark cycle). Ventilation was applied every $30 \mathrm{~min}$ and a quantitative pellet diet was provided at a fixed time each day. Animals passing the required routine tests including blood, urine, fecal, and electrocardiogram tests were selected for the experiment. These animals were fasted for $12 \mathrm{~h}$ prior to dosing and given free access to water.

The analytical method described above was applied to simultaneously determine the concentrations of doxifluridine, 5-FU, and 5-FUrd in the plasma from seven beagle dogs after oral administration of $200 \mathrm{mg}$ doxifluridine. Blood samples $(2 \mathrm{~mL})$ were collected from venous blood before $(0 \mathrm{~h})$ and at $0.25,0.5$, $0.75,1,1.25,1.5,2,2.5,3$, and $4 \mathrm{~h}$ after drug administration. No food was allowed until $4 \mathrm{~h}$ after dose administration and water intake was allowed until $2 \mathrm{~h}$ after dose administration. The blood samples were placed in heparinized tubes, and the plasma was separated by centrifugation at $3880 \times g$ for $10 \mathrm{~min}$. The study was conducted in compliance with the Good Laboratory Practice Regulations, Korea Food and Drug Administration (KFDA2005-79). Plasma concentration-time data of each analyte were analyzed with the non-compartmental method using WinNonlin software (standard edition 2.1; Pharsight Corp., Palo Alto, CA, USA).

\section{Results and Discussion}

Mass spectra. Full-scan product ion spectra for doxifluridine, 5-FU, 5-FUrd, and the IS (5-chlorouracil) contained precursor ions of $\mathrm{m} / \mathrm{z} 244.82,128.82,260.87$, and 144.76, respectively (Fig. 2). The most suitable collision energy (CE) was determined by observing the response obtained versus the selectivity response for fragment ions of each compound. The best CE sets were $-48.3 \mathrm{~V}$ for doxifluridine, $-26 \mathrm{~V}$ for 5 -FU, $-22 \mathrm{~V}$ for 5 FUrd, and $-30 \mathrm{~V}$ for IS, obtaining fragments of $\mathrm{m} / \mathrm{z} 41.90$ for doxifluridine, 41.24 for 5-FU, 128.42 for 5-FUrd, and 41.20 for the IS from each deprotonated compound.

Specificity and selectivity. Typical peak shapes and retention times of the MRM chromatograms are shown in Fig. 3. Doxifluridine, 5-FU, and 5-FUrd were clearly separated from interferences in the blank matrix under the current LC-MS/MS conditions. The chromatograms for five lots of blank plasma contained no endogenous peaks that co-eluted with the analytes or IS.

For the simultaneous determination of doxifluridine, 5-FU, 5-FUrd, and the IS, chromatographic conditions were developed to add selectivity to the validation and also to elute all four analytes with good peak shapes. The inclusion of $0.1 \%$ formic acid further improved the peak shape, and the optimal mobile phase consisted of $0.1 \%$ formic acid in 99:1 (v/v) MeOH: $\mathrm{H}_{2} \mathrm{O}$. The retention times of doxifluridine, 5-FU, 5-FUrd, and the IS in beagle dog plasma were about $8.7 \mathrm{~min}$, and the total runtime for each sample was about $12 \mathrm{~min}$.

Calibration curves. The plasma calibration curve was constructed using calibration standards of $0.05-10 \mu \mathrm{g} / \mathrm{mL}$ doxifluridine, $0.05-5 \mu \mathrm{g} / \mathrm{mL} 5$-FU, and $0.2-10 \mu \mathrm{g} / \mathrm{mL}$ 5-FUrd. The lowest limit of quantification (LLOQ) for doxifluridine, 5-FU, and 5 -FUrd were $0.05,0.05$, and $0.2 \mu \mathrm{g} / \mathrm{mL}$, respectively. The standard curve had reliable reproducibility across the calibration range. The calibration curve was prepared by determining the best fit of the peak area ratio (peak area analyte/peak area IS) versus concentration, and fit to $y=a x+b$ using a weighting factor $\left(1 / y^{2}\right)$. The linear coefficient of correlation for doxifluridine, 5-FU, and 5-FUrd was above 0.999 for five batches.

Precision and accuracy. The intra- and inter-assay accuracy and precision data, which were determined by analyzing five replicates of QC samples at four concentrations on 5 different days, are shown in Table 1. The accuracy (expressed as a percentage of nominal values) of the doxifluridine determination ranged from $93.06 \%$ to $110.94 \%$, with CVs of $3.97-5.75 \%$ and $1.53-3.50 \%$ for intra- and inter-day precision, respectively. The accuracy of 5-FU determination ranged from $92.87 \%$ to $103.62 \%$, and the intra- and inter-day precision ranged from

Table 1. Intra- and inter-assay accuracy and precision for the simultaneous determination of doxifluridine, 5-FU, and 5-FUrd in beagle dog plasma

\begin{tabular}{|c|c|c|c|c|c|}
\hline \multirow{2}{*}{ Analytes } & \multirow{2}{*}{ Concentration $(\mu \mathrm{g} / \mathrm{mL})$} & \multicolumn{2}{|c|}{ Intra-day $(n=5)$} & \multicolumn{2}{|c|}{ Inter-day $(n=25)$} \\
\hline & & Accuracy (\%) & CV (\%) & Accuracy $(\%)$ & CV $(\%)$ \\
\hline \multirow{4}{*}{ Doxifluridine } & 0.05 & 93.06 & 5.14 & 93.73 & 1.68 \\
\hline & 0.2 & 105.06 & 3.97 & 110.44 & 1.53 \\
\hline & 7.5 & 104.59 & 5.75 & 110.94 & 3.50 \\
\hline & 10 & 110.12 & 4.29 & 108.85 & 2.55 \\
\hline \multirow{4}{*}{ 5-FU } & 0.05 & 97.96 & 4.73 & 96.77 & 3.65 \\
\hline & 0.2 & 103.62 & 5.75 & 101.24 & 8.05 \\
\hline & 3 & 101.75 & 9.35 & 103.35 & 10.14 \\
\hline & 5 & 92.87 & 5.58 & 96.68 & 5.90 \\
\hline \multirow{4}{*}{ 5-FUrd } & 0.2 & 95.50 & 4.28 & 98.47 & 1.62 \\
\hline & 0.5 & 105.97 & 3.38 & 106.18 & 2.28 \\
\hline & 7.5 & 96.60 & 11.34 & 97.60 & 11.30 \\
\hline & 10 & 96.81 & 10.66 & 100.64 & 8.50 \\
\hline
\end{tabular}


$4.73 \%$ to $9.35 \%$ and $3.65 \%$ to $10.14 \%$, respectively. The accuracy of 5-FUrd determination ranged from $95.50 \%$ to $106.18 \%$, and the intra- and inter-day precision ranged from $3.38 \%$ to $11.34 \%$ and $1.62 \%$ to $11.30 \%$, respectively. These results demonstrated that the assay method was both reliable and reproducible.
Stability. A summary of the assay stability under various conditions is presented in Table 2. The mean integrated peak areas of the low and high QC samples were compared before and after the stability testing using procedures described in the Experimental section. Stock solutions were stable for 75 days at $-20{ }^{\circ} \mathrm{C}$. Doxifluridine, 5-FU, and 5-FUrd were stable in

(A)

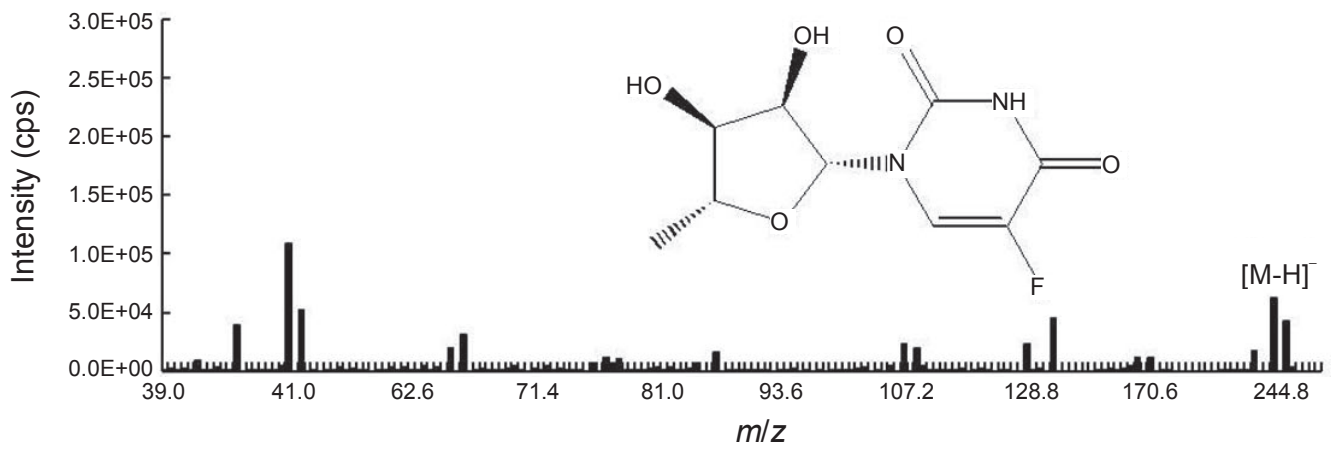

(B)

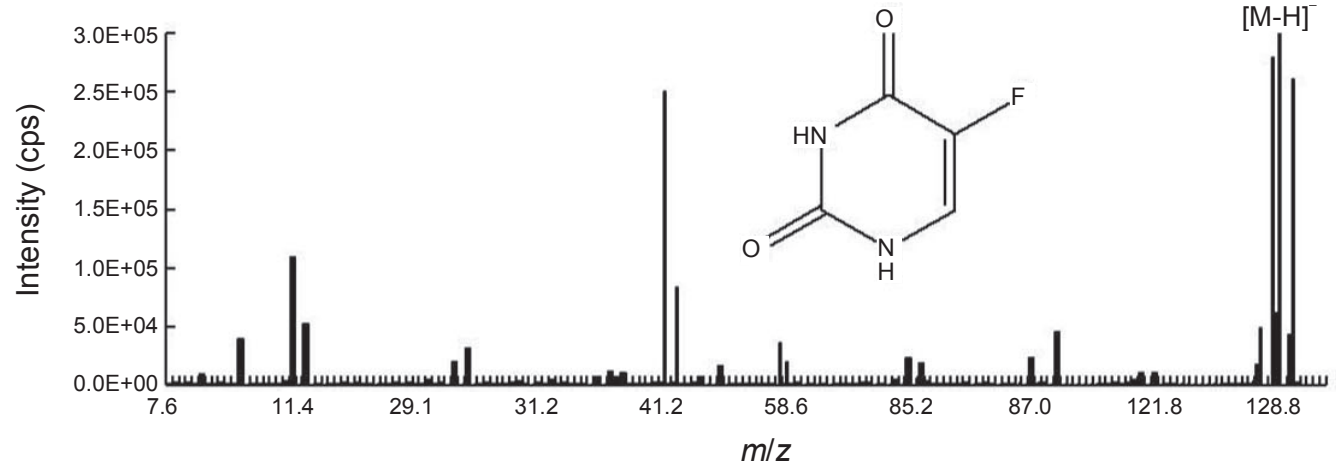

(C)

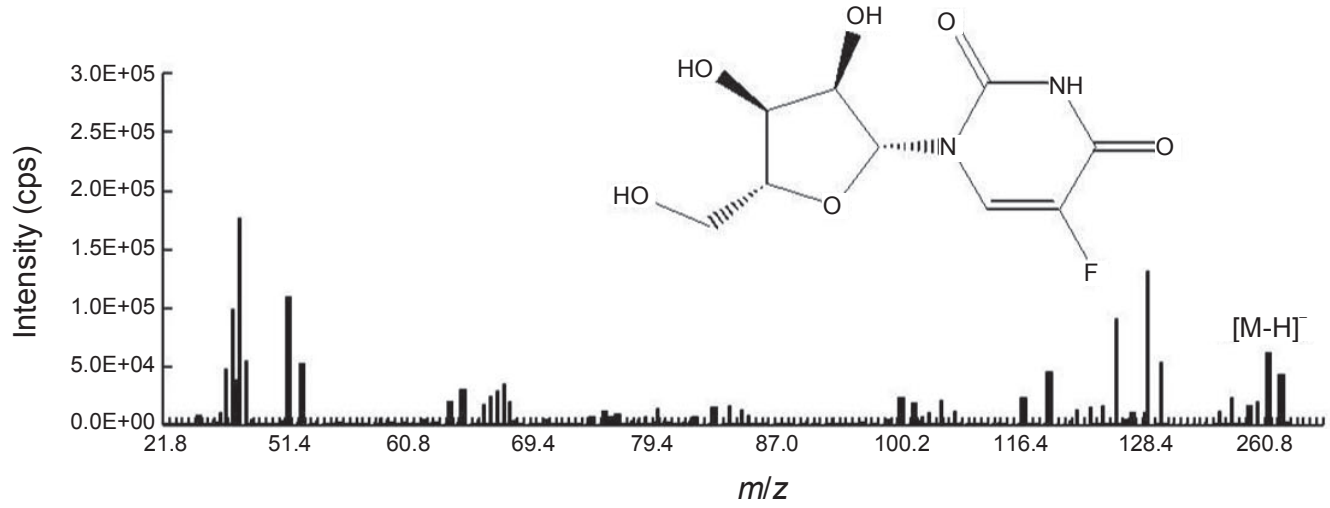

(d)

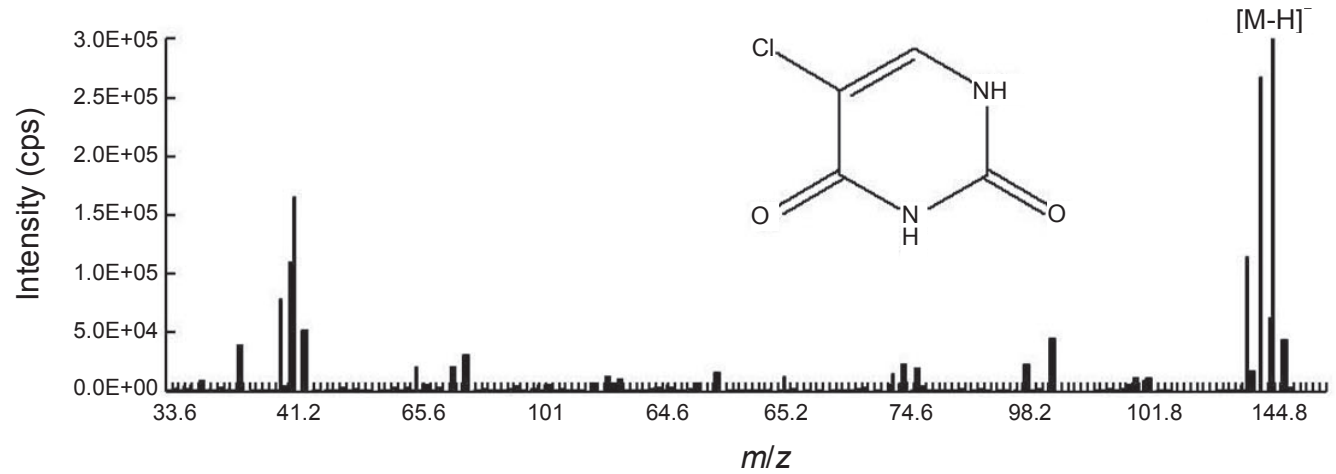

Figure 2. Tandem mass spectra show the ions obtained for (A) doxifluridine, (B) 5-FU, (C) 5-FUrd, and (D) 5-chlrouracil (internal standard, IS) using electrospray ionization in negative ion mode. 
(A)

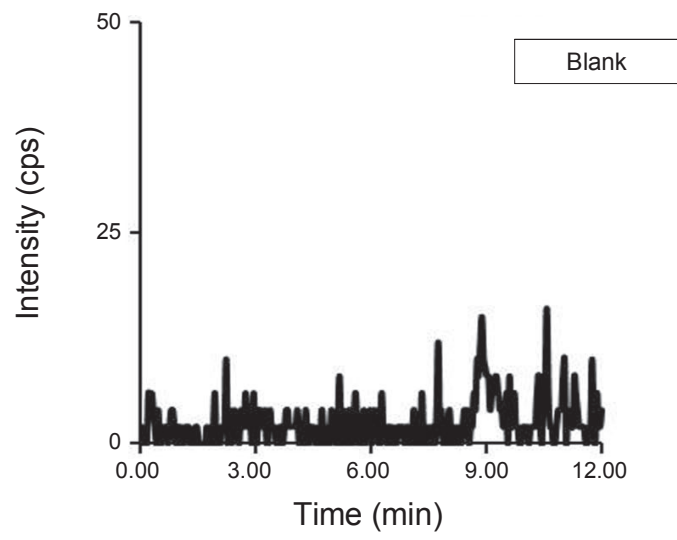

(B)

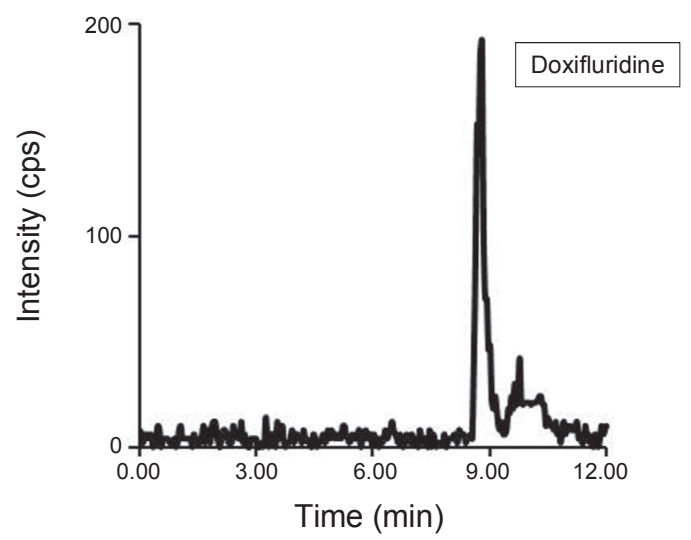

(C)

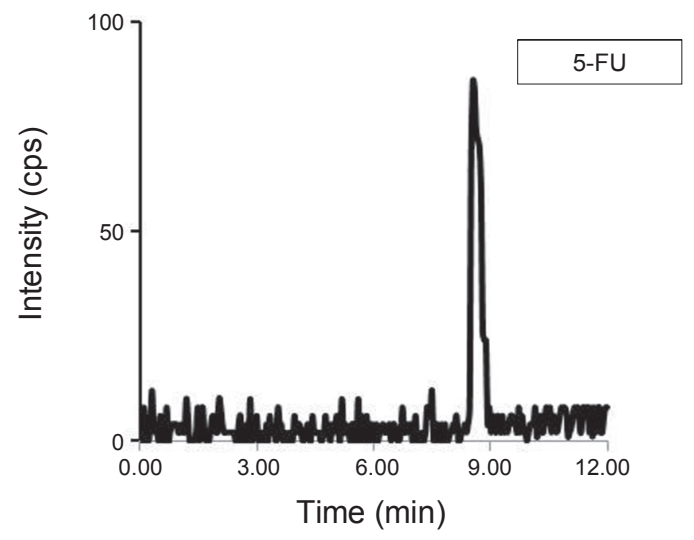

(D)

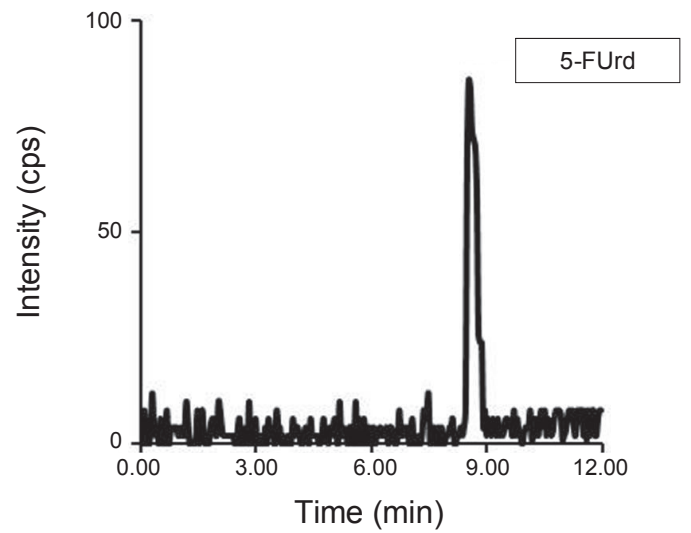

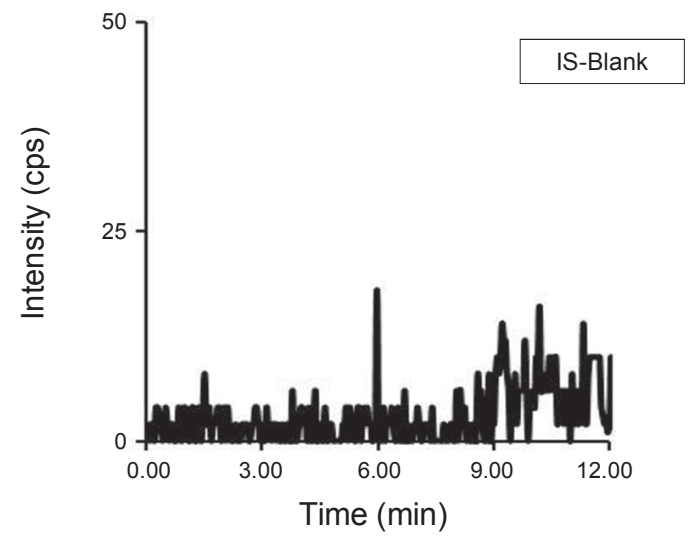
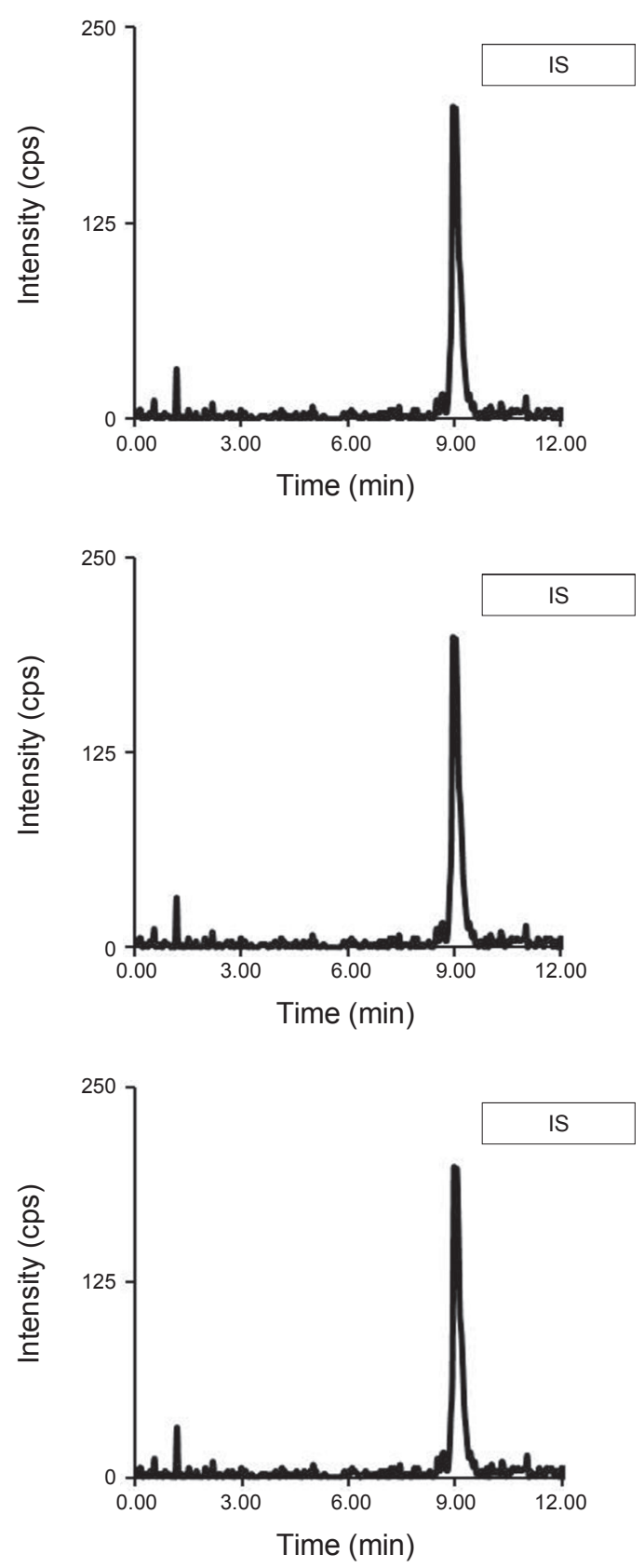

Figure 3. Typical MEM chromatograms of the analyte (left panel) and IS (right panel) in (A) blank plasma, (B) beagle dog plasma spiked with doxifluridine at LLOQ $(0.05 \mu \mathrm{g} / \mathrm{mL})$ and the IS, (C) beagle dog plasma spiked with 5-FU at LLOQ $(0.05 \mu \mathrm{g} / \mathrm{mL})$ and the IS, and (D) beagle dog plasma spiked with 5-FUrd at LLOQ $(0.2 \mu \mathrm{g} / \mathrm{mL})$ and the IS. 
Table 2. Stability of doxifluridine, 5-FU, and 5-FUrd

\begin{tabular}{|c|c|c|c|}
\hline Stability experiment & Storage condition & Stability $(\%)$ & Stability (\%) \\
\hline Doxifluridine & & $0.05 \mu \mathrm{g} / \mathrm{mL}$ & $10 \mu \mathrm{g} / \mathrm{mL}$ \\
\hline Short-term in plasma & Room temperature, for $6 \mathrm{~h}$ & 101.91 & 109.87 \\
\hline Process (extracted sample) & $4{ }^{\circ} \mathrm{C}$, for $24 \mathrm{~h}$ & 100.19 & 104.99 \\
\hline Freeze-thaw cycle in plasma & $-70{ }^{\circ} \mathrm{C}$, after third cycle & 92.82 & 97.26 \\
\hline Long-term in plasma & $-70{ }^{\circ} \mathrm{C}$, for 75 days & 93.62 & 101.10 \\
\hline Stock solution & $-20^{\circ} \mathrm{C}$, for 75 days & 94.82 & 103.95 \\
\hline $5-\mathrm{FU}$ & & $0.05 \mu \mathrm{g} / \mathrm{mL}$ & $5 \mu \mathrm{g} / \mathrm{mL}$ \\
\hline Short-term in plasma & Room temperature, for $6 \mathrm{~h}$ & 90.66 & 107.50 \\
\hline Process (extracted sample) & $4{ }^{\circ} \mathrm{C}$, for $24 \mathrm{~h}$ & 108.72 & 99.49 \\
\hline Freeze-thaw cycle in plasma & $-70{ }^{\circ} \mathrm{C}$, after third cycle & 102.51 & 105.30 \\
\hline Long-term in plasma & $-70{ }^{\circ} \mathrm{C}$, for 75 days & 108.61 & 99.96 \\
\hline Stock solution & $-20{ }^{\circ} \mathrm{C}$, for 75 days & 102.16 & 111.71 \\
\hline 5-FUrd & & $0.2 \mu \mathrm{g} / \mathrm{mL}$ & $10 \mu \mathrm{g} / \mathrm{mL}$ \\
\hline Short-term in plasma & Room temperature, for $6 \mathrm{~h}$ & 90.09 & 96.84 \\
\hline Process (extracted sample) & $4{ }^{\circ} \mathrm{C}$, for $24 \mathrm{~h}$ & 103.19 & 85.19 \\
\hline Freeze-thaw cycle in plasma & $-70{ }^{\circ} \mathrm{C}$, after third cycle & 112.64 & 106.98 \\
\hline Long-term in plasma & $-70{ }^{\circ} \mathrm{C}$, for 75 days & 95.88 & 88.10 \\
\hline Stock solution & $-20{ }^{\circ} \mathrm{C}$, for 75 days & 87.75 & 92.24 \\
\hline
\end{tabular}

Table 3. Pharmacokinetic parameters of doxifluridine, 5-FU, and 5FUrd in beagles after oral administration of $200 \mathrm{mg}$ doxifluridine $(n=$ 7)

\begin{tabular}{lccc}
\hline \multicolumn{1}{c}{ PK parameters } & Doxifluridine & 5-FU & 5-FUrd \\
\hline $\mathrm{C}_{\max }(\mu \mathrm{g} / \mathrm{mL})$ & $9.70 \pm 3.83$ & $0.53 \pm 0.20$ & $0.53 \pm 0.31$ \\
$\mathrm{~T}_{\max }(\mathrm{h})$ & $0.57 \pm 0.31$ & $1.00 \pm 0.52$ & $1.00 \pm 0.52$ \\
$\mathrm{AUC}_{\text {inf }}(\mu \mathrm{g} \cdot \mathrm{h} / \mathrm{mL})$ & $6.42 \pm 2.49$ & $0.29 \pm 0.12$ & $0.23 \pm 0.14$ \\
$\mathrm{t}_{1 / 2}(\mathrm{~h})$ & $0.34 \pm 0.13$ & $0.18 \pm 0.06$ & $0.31 \pm 0.18$ \\
Clearance $/ \mathrm{F}(\mathrm{L} / \mathrm{h})$ & $34.53 \pm 12.90$ & N.A. & N.A. \\
\hline
\end{tabular}

N.A.: not applicable

plasma after $6 \mathrm{~h}$ at room temperature, and no significant decreases of any of these compounds were detected after three freezethaw cycles. Doxifluridine, 5-FU, and 5-FUrd were stable in liquid/liquid extracted samples for $24 \mathrm{~h}$ at $4{ }^{\circ} \mathrm{C}$, and they were stable in plasma for 75 days at $-70^{\circ} \mathrm{C}$. Therefore, the short-term, long-term, freeze-thaw, and extraction stabilities were acceptable.

Recovery and matrix effects. The recovery was estimated by measuring the mean peak areas of the analytes in the extracted QC samples of $0.05,1$, and $10 \mu \mathrm{g} / \mathrm{mL}$ doxifluridine; $0.05,1$, and $5 \mu \mathrm{g} / \mathrm{mL} 5$-FU; and $0.2,2$, and $10 \mu \mathrm{g} / \mathrm{mL}$ for 5 -FUrd in five replicates, and then comparing them with those obtained from the extracted blank plasma samples post-spiked with the corresponding neat solutions in five replicates. The overall recovery rates for doxifluridine, 5-FU, and 5-FUrd were $92.78 \%, 84.67 \%$, and $89.76 \%$ with a precision (CV \%) of $7.21 \%, 1.89 \%$, and $3.27 \%$, respectively. The mean percentage recovery of $0.5 \mu \mathrm{g} /$ $\mathrm{mL}$ IS was $91.27 \%$ with $5.41 \%$ precision.

The matrix effect of doxifluridine, 5-FU, and 5-FUrd was

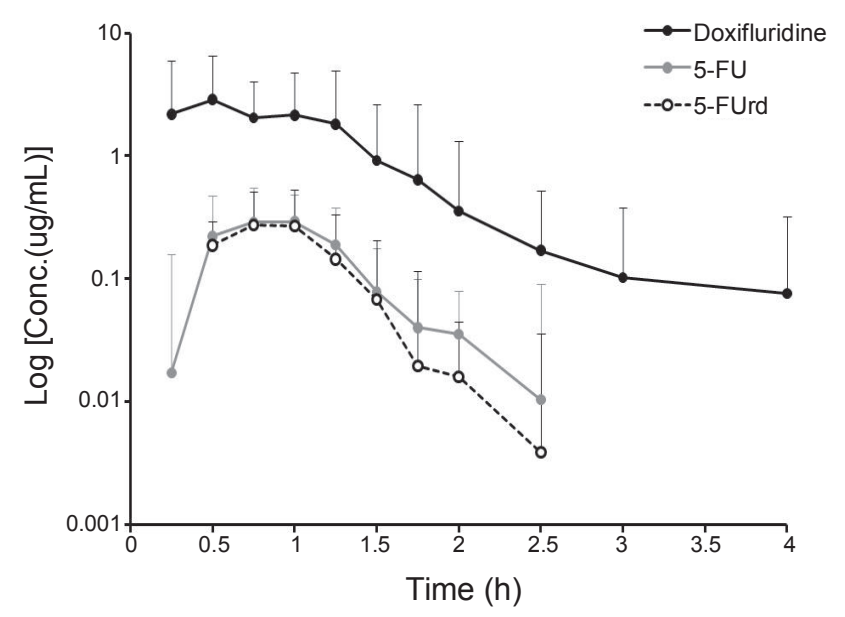

Figure 4. Mean plasma concentration versus time profile of doxiflu ridine and its two metabolites (5-FU and 5-FUrd) in beagles after oral administration of a single dose $(200 \mathrm{mg})$ of doxifluridine $(n=7)$.

assessed by comparing the peak area of the analytes in water (A) to that of a standard spiked sample preparation of blank plasma (B) to show that no ion enhancement or suppression occurred during the analysis. The average matrix effect values obtained were $3.47-12.14 \%$ for doxifluridine, $4.78-10.47 \%$ for 5-FU, and $4.24-11.04 \%$ for 5-FUrd. No significant peak area differences were observed. The matrix effect on the IS was $3.21 \%$ at $0.5 \mu \mathrm{g} / \mathrm{mL}$.

Pharmacokinetic studies. The analytical procedures described above were employed to quantify all analytes in plasma samples obtained from seven male beagle dogs that had been orally administered a single dose of $200 \mathrm{mg}$ doxifluridine. Profiles of the mean plasma concentration versus time are presented 
in Fig. 4. The non-compartmental pharmacokinetic parameters are given in Table 3.

After drug administration, the maximum concentration of doxifluridine in plasma $\left(\mathrm{C}_{\max }\right)$ was $9.70 \pm 3.83 \mu \mathrm{g} / \mathrm{mL}$ at $0.57 \pm$ $0.31 \mathrm{~h}$. The area under the plasma concentration versus time curve $\left(\mathrm{AUC}_{\mathrm{inf}}\right)$ for doxifluridine was $6.42 \pm 2.49 \mu \mathrm{g} \cdot \mathrm{h} / \mathrm{mL}$ and the half-life calculated from the terminal phase was $0.34 \pm$ $0.13 \mathrm{~h}$. The $\mathrm{C}_{\max }$ of 5-FU and 5-FUrd were $0.53 \pm 0.20 \mu \mathrm{g} / \mathrm{mL}$ at $1.00 \pm 0.52 \mathrm{~h}$ and $0.53 \pm 0.31 \mu \mathrm{g} / \mathrm{mL}$ at $1.00 \pm 0.52 \mathrm{~h}$, respectively. The $\mathrm{AUC}_{\text {inf }}$ values for 5-FU and 5-FUrd were $0.29 \pm$ $0.12 \mu \mathrm{g} \cdot \mathrm{h} / \mathrm{mL}$ and $0.23 \pm 0.14 \mu \mathrm{g} \cdot \mathrm{h} / \mathrm{mL}$, respectively. The halflife values for 5-FU and 5-FUrd determined from the terminal phase were $0.18 \pm 0.06 \mathrm{~h}$ and $0.31 \pm 0.18 \mathrm{~h}$, respectively.

\section{Conclusion}

This paper describes the validation of a bioanalytical LC-MS/ MS method for the simultaneous quantification of doxifluridine, 5-FU, and 5-FUrd using a single IS in beagle dog plasma. The selectivity, linearity, accuracy, precision, and stability of the method have been validated, and this is the first time that these three analytes were estimated simultaneously. This method for the quantification of doxifluridine and its two metabolites, 5-FU and 5-FUrd, in beagle dogs was applied successfully to the analysis of pharmacokinetic samples. The present method is a useful tool for pharmacokinetic studies of doxifluridine, 5-FU, and 5-FUrd.
Acknowledgments. This study was supported by a grant from the Korea Healthcare Technology R\&D Project, Ministry for Health, Welfare \& Family Affairs, Republic of Korea (A084144).

\section{References}

1. Bollag, W.; Hartmann, H. R. Eur. J. Cancer 1980, 16, 427.

2. Woo, Y. A.; Kim, G. H.; Jeong, E. J.; Kim, C. Y. J. Chromatogr. B Analyt. Technol. Biomed. Life Sci. 2008, 875, 487.

3. Joulia, J. M.; Pinguet, F.; Grosse, P. Y.; Astre, C.; Bressolle, F. J. Chromatogr. B Biomed. Sci. Appl. 1997, 692, 427.

4. Ishikawa, T.; Utoh, M.; Sawada, N.; Nishida, M.; Fukase, Y.; Sekiguchi, F.; Ishitsuka, H. Biochem. Pharmacol. 1998, 55, 1091.

5. Schüller, J.; Cassidy, J.; Dumont, E.; Roos, B.; Durston, S.; Banken, L.; Utoh, M.; Mori, K.; Weidekamm, E.; Reigner, B. Cancer Chemother. Pharmacol. 2000, 45, 291.

6. Pinedo, H. M.; Peters, G. F. J. Clin. Oncol. 1988, 6, 1653.

7. Ishitsuka, H.; Miwa, M.; Takemoto, K.; Fukuoka, K.; Itoga, A.; Maruyama, H. B. Gann. 1980, 71, 112.

8. Schaaf, L. J.; Dobbs, B. R.; Edwards, I. R.; Perrier, D. G. Eur. J. Clin. Pharmacol. 1988, 34, 439.

9. Yun, H. Y.; Bang, S. C.; Lee, K. C.; Baek, I. H.; Lee, S. P.; Kang, W.; Kwon, K. I. Talanta 2007, 71, 1553.

10. Rosing, H.; Man, W. Y.; Doyle, E.; Bult, A.; Beijnen, J. H. J. Liq. Chromatogr. Relat. Technol. 2000, 23, 329.

11. Dams, R.; Huestis, M. A.; Lambert, W. E.; Murphy, C. M. J. Am. Soc. Mass. Spectrom. 2003, 14, 1290.

12. Matuszewski, B. K.; Constanzer, M. L.; Chavez-Eng, C. M. Anal. Chem. 2003, 75, 3019. 